\title{
Tuberculosis in the European Union and European Economic Area: a survey of national tuberculosis programmes
}

\author{
Simon M. Collin (1) ${ }^{1}$, Gerard de Vries ${ }^{2}$, Knut Lönnroth ${ }^{3}$, Giovanni Battista Migliori $\mathbb{1}^{4}$, \\ Ibrahim Abubakar ${ }^{5}$, Sarah R. Anderson ${ }^{1}$ and Dominik Zenner ${ }^{5}$ \\ Affiliations: ${ }^{1}$ TB Unit, National Infection Service, Public Health England, London, UK. ${ }^{2}$ KNCV Tuberculosis \\ Foundation, Den Haag, The Netherlands. ${ }^{3}$ Karolinska Institutet, Stockholm, Sweden. ${ }^{4}$ Istituti Clinici Scientifici \\ Maugeri IRCCS, Pavia, Italy. ${ }^{5}$ UCL Institute for Global Health, London, UK.
}

Correspondence: Dominik Zenner, UCL Institute for Global Health, 30 Guilford Street, London, WC1N 1EH, UK. E-mail: dominik.zennerducl.ac.uk

@ERSpublications

There is a need for national TB control plans and investment in human resources to work towards TB elimination across the EU/EEA http://ow.ly/8gZG30m961Y

Cite this article as: Collin SM, de Vries G, Lönnroth K, et al. Tuberculosis in the European Union and European Economic Area: a survey of national tuberculosis programmes. Eur Respir J 2018; 52: 1801449 [https://doi.org/10.1183/13993003.01449-2018].

ABSTRACT How many European Union (EU) and European Economic Area (EEA) countries have national tuberculosis (TB) control plans/strategies, and what are the priority actions/populations and barriers to implementation?

In order to answer this question, a survey of EU/EEA national TB programme leads was undertaken.

The response rate was $100 \%$ ( 31 countries). 55\% of countries reported having a national TB strategy, all of which were in implementation; five countries were preparing a strategy. $74 \%$ had a defined organisational TB control structure with central coordination and $19 \%$ had a costed programme budget; few organisational structures included patient/civil society representation. The most frequently mentioned priority TB control actions were: reaching vulnerable population groups (80\%), screening for active TB in high-risk groups (63\%), implementing electronic registries (60\%), contact tracing and outbreak investigation (60\%), and tackling multidrug-resistant TB (60\%). Undocumented migrants were the most commonly (46\%) identified priority population. Perceived obstacles to implementation included barriers related to care recipients (lack of TB knowledge, treatment seeking/adherence), care providers (including need for specialist training of nurses and doctors) and health system constraints (funding, communication between healthcare and social care systems).

This survey has provided an insight into TB control programmes across the EU/EEA that will inform the development of a TB strategy toolkit for member states. 


\section{Introduction}

Tuberculosis (TB) incidence continues to decline across the European Union (EU) and European Economic Area (EEA), but projected trends show that a strengthening of efforts is needed if the World Health Organization (WHO) End TB vision is to be realised by EU/EEA member states [1, 2]. Owing to the specific TB epidemiology in low-incidence countries, programmes to work towards TB elimination in this context typically include interventions directed at vulnerable and high-risk groups alongside wider health system efforts to improve treatments, prevent resistance and implement new technologies [2-5]. The WHO End TB Strategy [6] recommends that each country develops a national TB control plan or strategy [7]. Across the EU/EEA, support for national TB programmes is provided at the supranational level through the European Centre for Disease Prevention and Control (ECDC) and WHO/Europe, taking into account social, economic and epidemiological heterogeneity between and within member states [1]. One of the core indicators of the ECDC Framework Action Plan (2008) was the availability of a national TB control plan or strategy formally adopted by the respective national government [8]. A 2013 survey of European countries found that only 15 EU/EEA countries had a national TB control plan [9], although this survey pre-dated publication of the WHO End TB Strategy.

Here we report the findings of a survey that gathered information from national TB programme leaders across the EU/EEA regarding the availability, implementation and content of national TB control plans. The aim of the study was to obtain an up-to-date picture of national TB control plans and strategies, including prioritisation of action areas and barriers to the implementation of interventions for TB control and prevention.

\section{Methods}

This survey was conducted as part of the European Commission-funded E-DETECT TB (Early Detection and Integrated Management of Tuberculosis in Europe) project (https://e-detecttb.eu), which aims to improve TB control efforts across Europe through translational research designed to reach high-risk groups in EU/EEA countries as well as the development of a practical toolkit to support national TB strategy development and implementation [10].

The survey used an online questionnaire to collect data from national $\mathrm{TB}$ programme leaders or representatives across all 31 countries of the EU/EEA. The questionnaire was designed and tested at Public Health England (London, UK) in collaboration with E-DETECT TB partners. The survey comprised 11 sections (table 1), including fixed and open questions (supplementary material file S1), and was implemented using SelectSurvey (SelectSurvey.NETv4; ClassApps, Kansas City, MO, USA). The methodology was consistent with that of a previous European survey [9], thereby allowing progress on some indicators to be evaluated.

The barriers section was aligned with the SURE (Supporting the Use of Research Evidence) checklist of barriers to implementing health policy options [11], thereby generating a list of 44 factors that could impede TB control and prevention. These were grouped under four subheadings: recipients of care (six factors), providers of care (five factors), health system constraints (27 factors), and social and political constraints (six factors). Invitations to complete the survey were distributed via e-mail to national $\mathrm{TB}$

TABLE 1 The 11 sections in the E-DETECT TB survey of European Union/European Economic Area national tuberculosis (TB) programme leaders

\section{National strategies, coordination and resources}

1 Availability and implementation of a national TB control plan or strategy

2 TB programme coordination and stakeholder representation

3 Resources (including budget, workforce development, and new tools for TB control and prevention)

$4 \quad$ Monitoring and surveillance

\section{Finding and treating TB in the population}

5 TB in high-risk and vulnerable population groups

6 Clinical and technical (guidelines, laboratory quality assurance, contact tracing, vaccination)

7 MDR-TB

$8 \mathrm{HIV} / \mathrm{TB}$

\section{Priorities and barriers}

9 Priority actions: which existing or new actions are most important or have the greatest urgency?

10 Priority populations: which groups have the highest unmet need for TB detection and treatment?

11 Barriers to TB control and prevention

MDR: multidrug-resistant. 
programme managers listed by WHO and ECDC on May 17, 2017. Survey questions were worded in accordance with terminology used in ECDC documents [8] that are familiar to EU/EEA TB programme teams. In addition, the survey administrator (S.M. Collin) was available by e-mail, telephone and at the 2017 Wolfheze Workshops (The Hague, The Netherlands) in case clarification was needed.

Data were exported from SelectSurvey to Stata release 13 (StataCorp, College Station, TX, USA). Data were analysed descriptively, tabulating frequency (percentage) of responses for each questionnaire item for all countries combined and separately for low ( $<10$ per 100000) and medium/high ( $\geqslant 10$ per 100000) TB incidence [1] countries. Questionnaire items that asked respondents to rate priorities or unmet need as high/medium/low were given an overall percentage score, which was calculated by dividing a weighted sum of individual responses (coded as low $=0$, medium $=1$ and high=2) by the theoretical maximum score.

\section{Results}

\section{Survey completion}

The survey was completed by programme managers or their delegated representatives in all of the 31 countries (100\% response rate). Responses were received between May 17 and September 19, 2017. Responses for Liechtenstein were provided jointly by representatives from Liechtenstein and Switzerland (a non-EU/EEA country), reflecting a unified approach to TB control and prevention in the two countries. We retained $\mathrm{N}=31$ as the denominator for our analyses (not all questions were mandatory, hence some responses show a denominator $\mathrm{N}<31$ ). Survey responses not quantified in table 2 or in the text are summarised in the tables in supplementary material file S2.

\section{National strategies, coordination and resources \\ Availability and implementation of a national TB control plan or strategy}

Just over half (17 out of 31) of EU/EEA countries had a national TB control plan or strategy (figure 1 and table 2). All of those with a plan had begun implementation, including six in 2015/2016 and five in 20112013. 13 plans covered periods of 3-9 years duration (median 5 years), typically beginning in 2011-2016 and ending in 2017-2020, and the remainder were open-ended (four plans). Of the 14 countries without a national TB control plan or strategy, five were either preparing a plan or intended to prepare a plan, three of which were expected to be finalised by 2019 .

\section{TB programme coordination}

Three-quarters (23 out of 31 ) of countries reported having a clearly defined organisational structure and about half of these were defined in the national TB control plan or strategy (table 2). TB control and prevention was coordinated centrally by a national TB control board, committee or other formal body in

TABLE 2 Availability and implementation of a national tuberculosis (TB) control plan/strategy, and TB programme coordination in European Union/European Economic Area countries

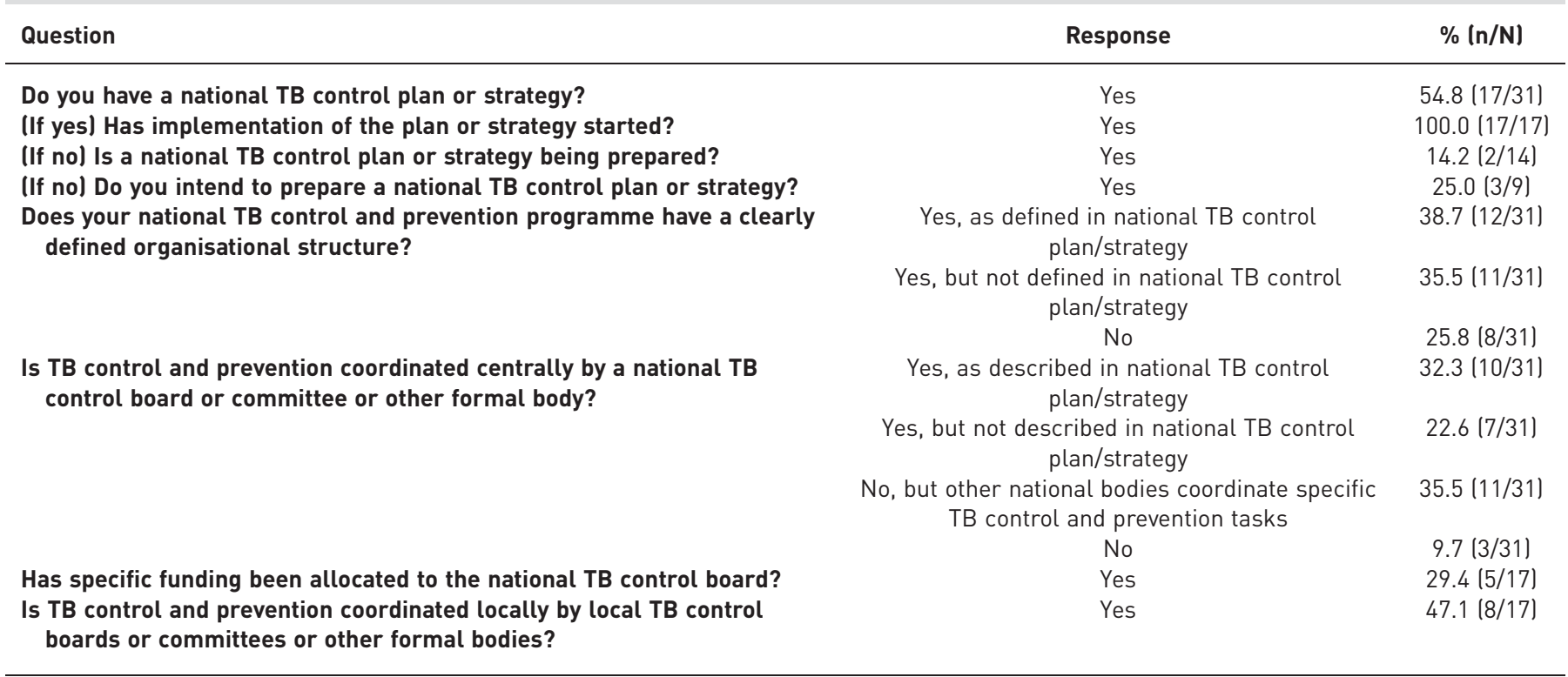




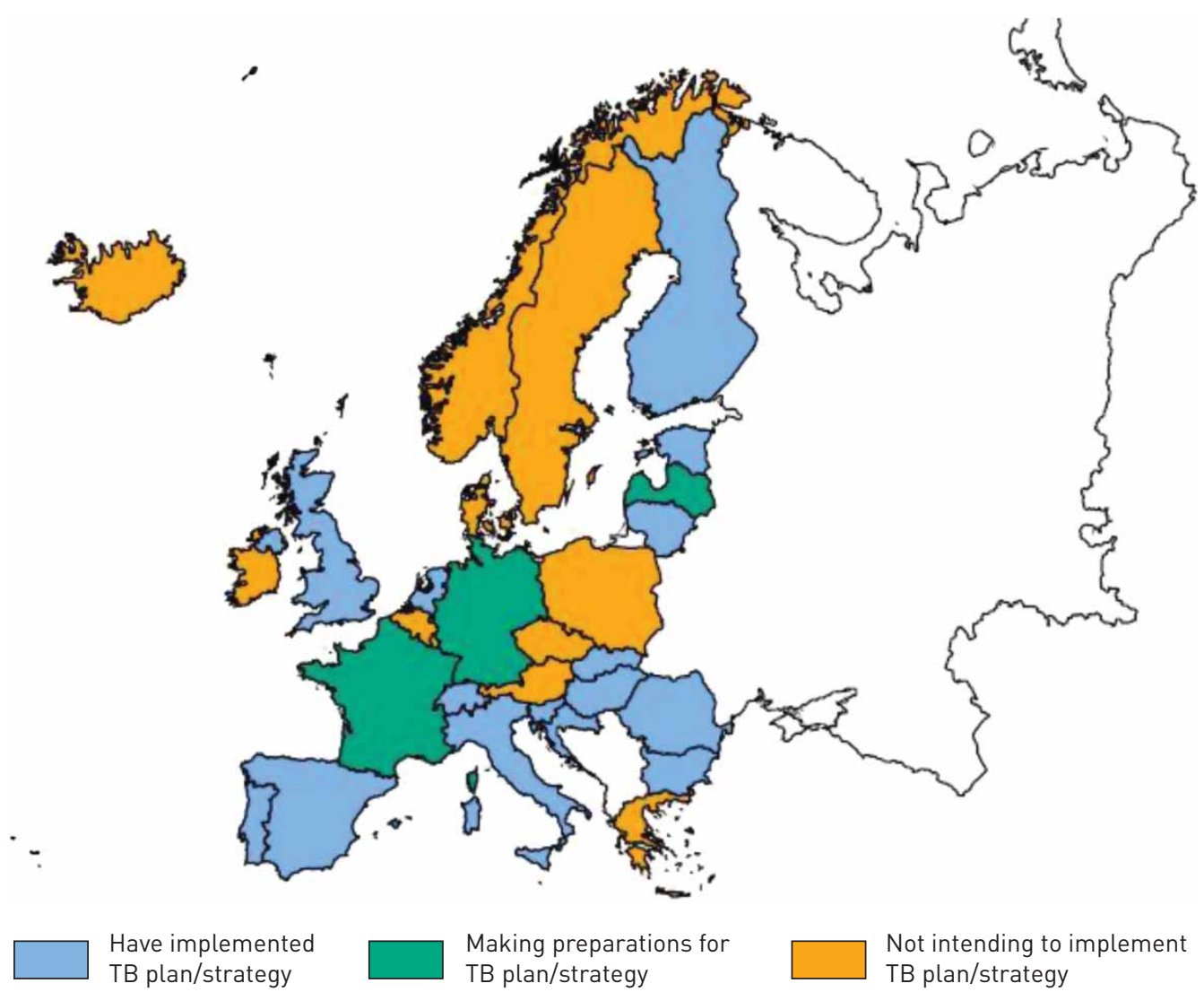

FIGURE 1 National tuberculosis (TB) control plans or strategies in European Union/European Economic Area countries.

$55 \%$ (17 out of 31 ) of countries and by other national bodies or regional control structures in $35.5 \%$ (11 out of 31) of countries.

\section{Stakeholder representation}

The majority $(>80 \%)$ of the 17 national TB control boards (or committees or other formal bodies) had Ministry/Dept of Health and national public health department representation. More than half had clinical, laboratory, epidemiology and local public health representatives, but professional bodies (41\%), local TB control boards (35\%) and nursing (29\%) were less frequently represented. Nongovernmental organisations (NGOs), patients and civil society were represented on few $(n<5)$ control boards, as were pharmacies $(n=2)$ and private health providers $(n=1)$. Two of the 17 coordinating bodies met monthly, five met annually, five met irregularly and the remainder met every 3-6 months. Specific funding had been allocated to five out of 17 coordinating bodies and five out of 17 received regular reports from local (regional, district, state, provincial, etc.) TB control boards, committees or other formal bodies.

\section{Budget}

One-fifth of countries (six out of 31) had a costed budget for a national TB programme, with 17 of the remaining 25 countries having budgets for parts of a TB programme and/or providing for TB-related activities within their national, federal or municipal healthcare systems. Half of countries (16 out of 31) had conducted an impact assessment or other financial or health economic assessment of the likely impact of TB control.

\section{Workforce training and development}

One-fifth of countries had a strategy for training and developing a specialist TB workforce (coordinated at a national level in 14 out of 16 countries). The parts of the TB workforce with the greatest perceived need for training and development were: community/primary health workers (59.3\% (16 out of 27)), specialist nurses $(51.9 \%$ (14 out of 27$)$ ), specialist doctors (37.0\% (10 out of 27$)$ ), microbiologists (33.3\% (nine out of 27)), epidemiologists (33.3\% (nine out of 27)) and surveillance scientists (18.5\% (five out of 27)). Five countries mentioned other needs for workforce training and development, including: general practitioners 
(three countries), radiologists, pulmonologists and specialist radiologists (one country), and immigration and prison services (one country). Five respondents commented that low TB incidence in their country led to low knowledge and experience of TB because healthcare professionals encountered few cases; a further two stated that there was a need for knowledge updates among healthcare workers and general practitioners.

\section{New tools for TB control and prevention}

One-fifth of countries had a strategy for introducing and implementing new tools for TB control and prevention, including: rapid diagnostic tests $(85.7 \%$ (12 out of 14$))$, treatment observation $(71.4 \%$ (10 out of 14)), infection control (57.1\% (eight out of 14)), drug susceptibility testing (57.1\% (eight out of 14)), surveillance $(50.0 \%$ (seven out of 14$)$ ), microbiology (50.0\% (seven out of 14$)$ ), contact tracing (35.7\% (five out of 14)) and outbreak investigation (28.6\% (four out of 14)).

\section{Monitoring and surveillance}

All countries had a national TB case registry, and three-quarters (23 out of 31 ) had a strategy for monitoring and evaluation of TB control and prevention, half of which (11 out of 23) were documented in the national TB control plan/strategy. The median (range) number of full-time equivalent staff assigned to TB surveillance in national offices was $2(0.5-8.5)$.

\section{Finding and treating TB in the population}

Raising awareness of TB

$21(67.7 \%)$ countries had programmes for raising awareness of TB at the community or primary care level, of which four were documented in the national TB control plan/strategy. The community or primary care groups reached by these programmes included: primary care doctors/general practitioners (95.2\% (20 out of 21$)$ ), primary care health workers $(76.2 \%$ (16 out of 21$)$ ), social workers (66.7\% (14 out of 21$)$ ) and the general public (57.1\% (12 out of 21)). Other groups for awareness raising included: services for people living with HIV, prisons, schools and NGOs working with vulnerable groups.

\section{Active and latent TB in high-risk and vulnerable groups}

A number of vulnerable population groups were identified as being at higher risk of having latent or active TB: asylum seekers (88.5\%), prisoners (84.6\%), refugees (76.9\%), documented migrants (69.2\%), undocumented migrants $(57.7 \%)$ and people with drug problems (57.7\%). Screening for active TB was conducted among asylum seekers in $77.4 \%$ of countries, refugees in $71.0 \%$ of countries and current prisoners in $74.2 \%$ of countries. Point-of-entry and post-entry screening for active TB among documented migrants was conducted by $32.3 \%$ and $41.9 \%$ of countries, respectively. Two countries conducted screening for active $\mathrm{TB}$ in social care institutions and for people entering shared community accommodation. A strategy for TB control in prisons was in place in $77.4 \%$ of countries, of which nine were documented in the national TB control plan/strategy. The groups most commonly identified for latent TB infection (LTBI) screening were: asylum seekers (33.3\%), refugees (26.7\%) and current prisoners (26.7\%). Point-of-entry and post-entry screening for LTBI in documented migrants was conducted by $20.0 \%$ and $16.7 \%$ of countries, respectively.

\section{Contact tracing}

Contacts of cases were tested for LTBI in $86.7 \%$ (26 out of 30 ) of countries, and the majority (83.9\% (26 out of 31)) had a strategy to implement and ensure comprehensive contact tracing, of which half (13 out of 26) were documented in a national $\mathrm{TB}$ control plan/strategy. Recommended approaches to tracing included: household contacts (96.2\% (25 out of 26)), workplace contacts (92.3\% (24 out of 26)), healthcare facility contacts $(92.3 \%$ (24 out of 26$))$ and community contacts $(80.8 \%$ (21 out of 26$))$.

\section{Vaccination}

Two-thirds of countries (20 out of 30) had a strategy to provide and promote bacille Calmette-Guérin (BCG) vaccination, of which half (nine out of 20) were documented in the national TB control plan/ strategy. The proportions of BCG vaccination strategies that included universal infant, high-risk infant and high-risk adult BCG vaccination were $42.1 \%$ (eight out of 19 ), $57.9 \%$ (11 out of 19 ) and $21.1 \%$ (four out of 19), respectively. Of the 10 countries that did not have a BCG vaccination strategy, two countries vaccinated infants born to immigrant parents from high $\mathrm{TB}$ incidence countries and three countries vaccinate selectively in high-risk situations.

Multidrug-resistant and extensively drug-resistant TB

The majority of countries ( $80 \%$ (24 out of 30 )) had a strategy to tackle drug-resistant TB, of which $58 \%$ (14 out of 24) were documented in the national TB control plan/strategy. Measures to tackle drug-resistant 
TB included: using directly observed treatment (87.5\% (21 out of 24 , including video-observed in four out of 21)), patient-centred multidrug-resistant (MDR)-TB case management (79.2\% (19 out of 24)), concentrating expertise in MDR-TB treatment centres (79.2\% (19 out of 24)), infection control in health facilities $(75.0 \%$ (18 out of 24)) and multidisciplinary MDR-TB case management (66.7\% (16 out of 24$)$ ). All of the countries with a drug-resistant TB strategy routinely conducted first-line drug susceptibility testing.

\section{HIV/TB}

An integrated approach to TB and HIV control was reported by $61.3 \%$ (19 out of 31 ) of countries, of which 58\% (seven out of 19) were documented in the national TB control plan/strategy. TB patients were routinely tested for HIV in $77.4 \%$ (24 out of 31 ) of countries and people living with HIV were screened for TB in $74.2 \%$ (23 out of 31 ) of countries; $61.2 \%$ (19 out of 31 ) of countries did both. The majority of countries $(80.7 \%$ (25 out of 31$)$ ) monitored TB/HIV co-infection at the national level.

\section{Guidelines and professional networks}

National TB control and prevention guidelines were available in $80.7 \%$ (25 out of 31 ) of countries and laboratory diagnostic services were subject to external quality assurance in all countries. Forms of professional and clinical support available to clinicians included: clinical guidelines (90.3\% (28 out of 31)), specialist training $(74.2 \%$ (23 out of 31$)$ ), infection control guidelines (74.2\% (23 out of 31$)$ ), clinical networks $(54.8 \%$ (17 out of 31$))$, research meetings (45.2\% (14 out of 31$)$ ) and local multidisciplinary teams (41.9\% (13 out of 31)). Expert group meetings for clinicians managing difficult and MDR- or extensively drug-resistant (XDR)-TB cases were mentioned by four out of 31 respondents.

\section{Priorities and barriers}

Priority actions: "In relation to TB control in your country, which existing or new actions do you think are most important or have the greatest urgency?"

Of the 18 pre-specified action areas, the five most frequently rated as high priority were: reaching vulnerable population groups (80.0\%), screening for active TB in high-risk population groups (63.3\%), implementing electronic TB case registries (60.0\%), contact tracing and outbreak investigation (60.0\%), and MDR-TB (60.0\%). The two most frequently rated as low priority were: BCG vaccination (56.7\%) and establishing or managing local TB control boards (43.3\%). Several countries indicated other high-priority action areas, including: mobile outreach, increasing TB expertise and experience in healthcare professionals, and broader social support for vulnerable groups. Weighted scores based on high-, mediumand low-priority ratings are show in figure 2.

Priority populations: "In relation to TB control in your country, which population groups do you think have the highest unmet need for TB detection and treatment?"

Respondents most frequently identified a high level of unmet need for TB detection among undocumented migrants (46.7\%); unmet need for TB detection was rated as low or medium among other vulnerable/ high-risk groups. Unmet need for TB treatment was ranked as medium/high for homeless people by $61.3 \%$ of countries and for undocumented migrants by $66.7 \%$ of countries. Weighted scores based on high, medium and low ratings of unmet need are show in figure 3.

Barriers: "Which of the following factors impede TB control in your country?"

Three barriers among service users were identified by a majority of countries: people in vulnerable/ high-risk groups lacking knowledge about TB (74.2\%), low motivation to adhere to treatment among vulnerable/high-risk groups (70.0\%) and low motivation to seek treatment among vulnerable/high-risk groups (58.1\%) (figure 4). Only one "provider of care" factor was identified by a majority of countries: the need for specialist training for nurses in TB patient care $(56.7 \%)$. "Social and political" constraints were perceived by only 19-36\% of countries as impeding TB control. Four health system constraints were selected by $>40 \%$ of respondents: numbers of specialist TB nurses (45.2\%), funding of national TB control and prevention programme $(43.3 \%)$, funding in the wider healthcare system $(43.3 \%)$, and communication between the healthcare and social care systems (41.9\%) (figure 5).

\section{Responses to key items in relation to national TB incidence}

10 EU/EEA countries had TB incidence $\geqslant 10 / 100000$ according to ECDC estimates for 2006-2015: the UK (10 per 100000), Spain (12 per 100000$)$, Croatia (13 per 100000), Estonia (18 per 100000), Poland (19 per 100000), Portugal (23 per 100000$)$, Bulgaria (24 per 100000$)$, Latvia (41 per 100000), Lithuania (56 per 100000 ) and Romania (84 per 100000 ). Of these 10 countries, $80 \%$ had a national TB control plan/strategy compared with $43 \%$ of countries with low TB incidence (table 3 ). In terms of total annual numbers of incident TB cases (based on 2015 data), two countries with no national plan/strategy had 


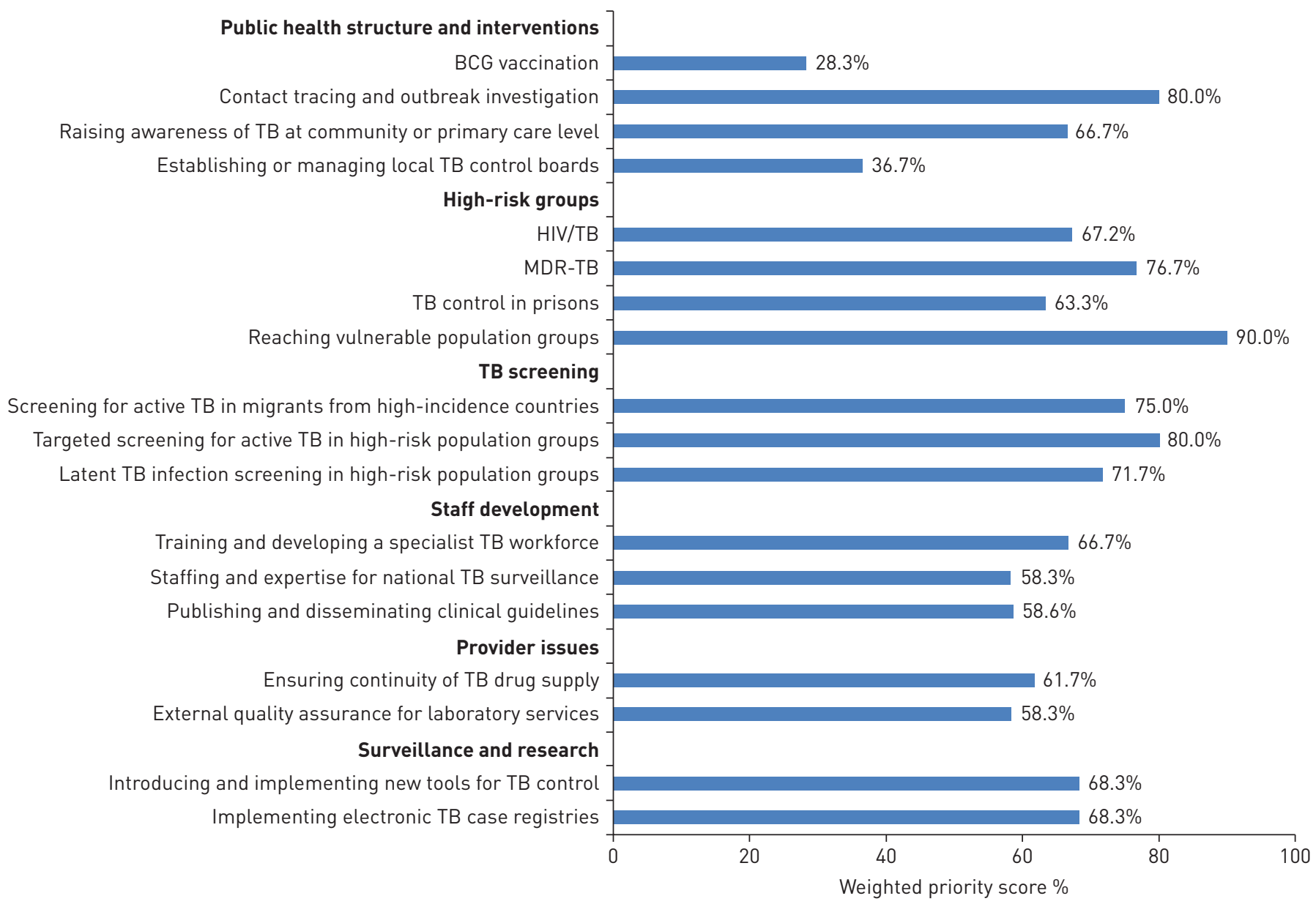

FIGURE 2 Priority actions: "In relation to TB control in your country, which existing or new actions do you think are most important or have the greatest urgency?" TB: tuberculosis; BCG: bacille Calmette-Guérin; MDR: multidrug-resistant. Priority actions were ranked "low", "medium" and "high" priority by respondents. We scored these responses low=0, medium=1 and high=2, and converted the total score for each priority action into a percentage by dividing the total by the maximum possible score $[=62$ if all 31 countries indicated "high" priority).

$<50$ cases, three countries had 300-500 cases, four countries had 500-800 cases and four countries had $>1000$ cases. All countries with medium/high TB incidence had a clearly defined organisational structure and central coordination of $\mathrm{TB}$ control compared with $62 \%$ and $86 \%$, respectively, in low-incidence countries. There were no differences in the proportions with central coordination or specific funding for TB control.

A higher proportion of countries with low TB incidence identified screening for active TB in migrants from high-incidence countries as a high-priority action (67\% compared with $30 \%$ of countries with medium/high TB incidence), whereas medium/high TB incidence countries were more likely to identify MDR-TB and HIV/TB as high priority (table 3). Differences in responses to the question about unmet need for TB detection and treatment were apparent only for TB detection in undocumented migrants (62\% of low-incidence countries reported high unmet need compared with $11 \%$ of medium/high-incidence countries) and TB treatment in people with alcohol problems (40\% of medium/high-incidence countries reported high unmet need compared with $10 \%$ of low-incidence countries). The median number of factors identified as barriers to TB control and prevention were similar in low- and medium/high-incidence countries (table 3 ).

\section{Discussion}

This survey has provided an up-to-date picture of the availability, implementation and content of national TB control plans in EU/EEA countries, and insights into priority action areas, population groups and barriers to programme implementation. It shows that just over half of EU/EEA countries had a national TB strategy, of which all had been or are being implemented. Although the majority of countries had a defined organisational structure and half had central coordination, a minority had a costed programme 


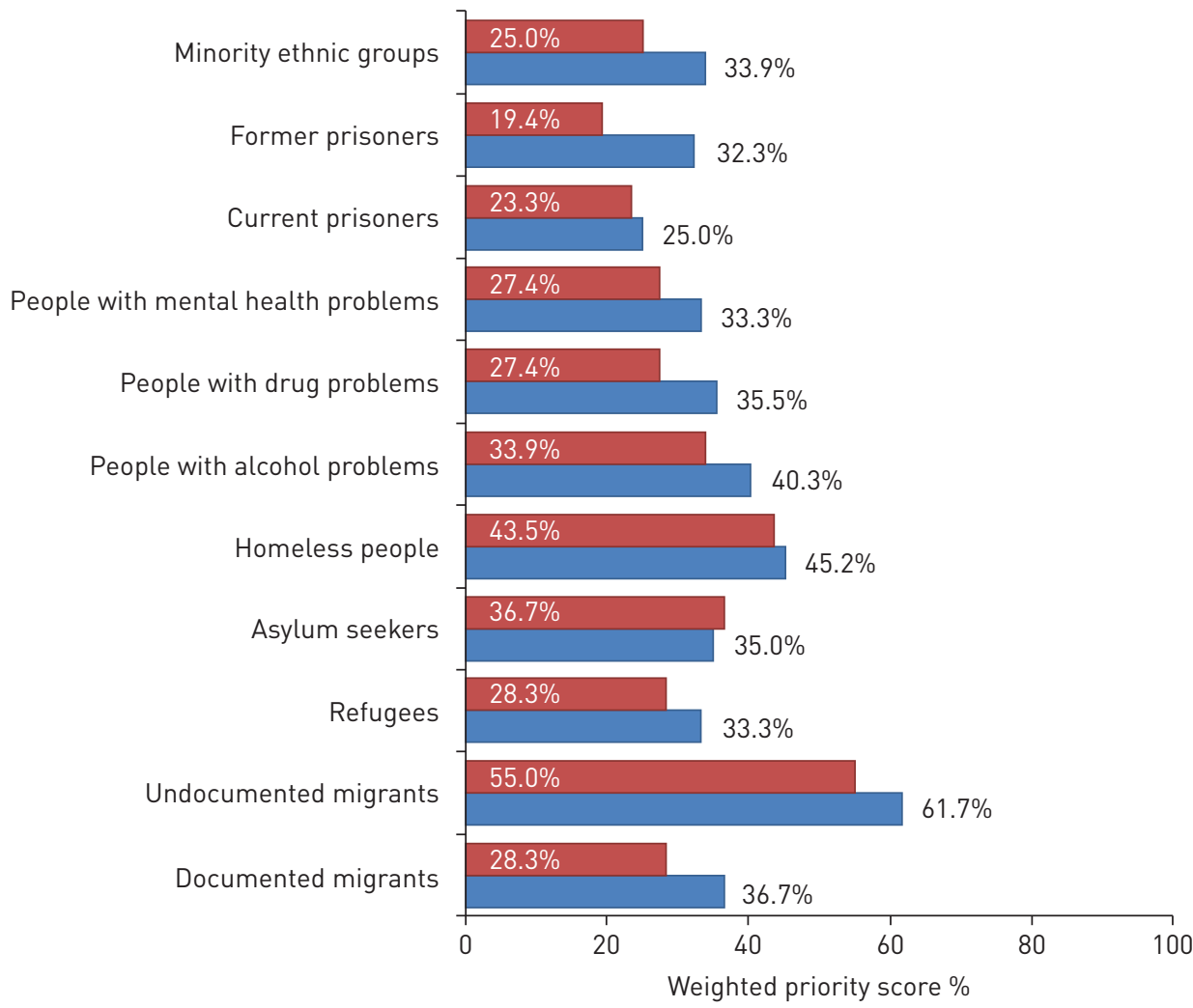

TB treatment priority $\square$ TB detection priority

FIGURE 3 Priority populations: "In relation to TB control in your country, which population groups do you think have the highest unmet need for TB detection and treatment?" TB: tuberculosis. Unmet need was ranked "low", "medium" and "high" by respondents. We scored these responses low=0, medium=1 and high=2, and converted the total score for each priority population into a percentage by dividing the total by the maximum possible score (=62 if all 31 countries indicated "high" unmet need).

budget, suggesting suboptimal capacity to coordinate activities at the national level $[12,13]$. Of note is that few national TB control boards included patient or civil society representatives.

A majority of respondents mentioned vulnerable population groups, screening for active TB in high-risk groups, implementing electronic case registries and MDR-TB as priority actions. These were selected by respondents from a list of 18 action areas, which we specified under the tacit assumption that they are not "more important" than ensuring the fundamentals of TB diagnosis and treatment within a universal healthcare system or guaranteeing social protections and minimum socioeconomic conditions to prevent TB on a societal level [14]. Rather, they represent specific areas for new or scaled-up interventions as part of an overall strengthening of efforts to control and prevent TB. As might be expected, a higher proportion $(62 \%)$ of low TB incidence countries identified undocumented migrants as having high unmet need for TB detection and treatment than did low/medium TB incidence countries (21\%), reflecting the disproportionately high number of TB cases occurring in migrant groups in low TB incidence countries [2].

One-third of respondents indicated a lack of government recognition of TB control as a public health priority, but the most commonly cited barriers related to recipients of care, care providers (mainly specialist training) and health system constraints. The perception that TB control can be impeded by factors related to recipients of care, i.e. lack of $\mathrm{TB}$ knowledge, seeking care and adherence, must be interpreted as a challenge to providers to address issues of awareness and stigma [15], and to develop and deploy evidence-based interventions [16]. The importance of good communication and coordination within the healthcare system and between the health and social care systems has been demonstrated in reports of cases and outbreaks in EU/EEA countries [17, 18].

\section{Survey findings in the context of other studies}

A 2013 survey of 38 European national TB programme representatives found that, of 26 countries also included in our survey, 15 out of the 26 had a national TB control plan [9]. In our study this proportion was unchanged, but three additional countries now indicated that they had a plan (for 2007-2016, 


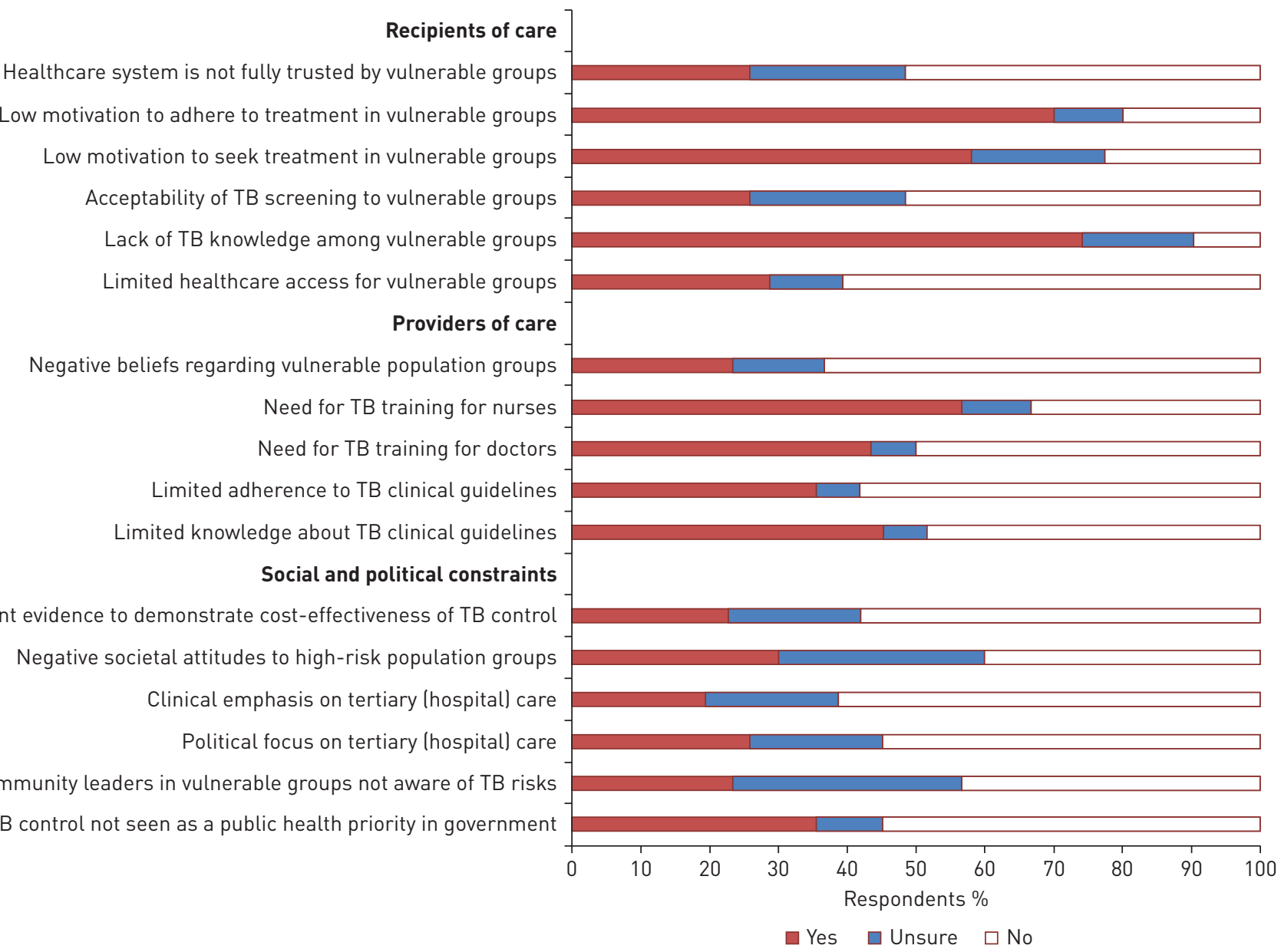

FIGURE 4 Barriers: "Which of the following factors impede TB control in your country?" TB: tuberculosis.

2013-2018 and 2015-2020) and three countries that previously had a plan responded as follows: one had a formalised TB programme during 2007-2009 that was finished to limit the number of vertical plans and committees in public health, although the framework was still in place and a new programme was planned for 2017-2020; one had a federal structure with legally established local, regional and national responsibilities and a coherent approach to TB control and prevention that was considered to replace the need for a national programme; and one had a plan scheduled for 2019-2021. The availability of a (costed) national TB control plan that has been formally adopted by the national government was one of the core indicators for the Framework Action Plan to Fight TB in the EU [8]. Our survey results indicate that two-thirds (20 out of 31) of EU/EEA countries will have implemented a national TB control plan before 2020 .

The single most important priority for stakeholders was TB control among vulnerable, particularly migrant populations. This perception of need may in part be attributed to recent experience of large refugee movements across continental Europe. Barriers to accessing services [19] and the large numbers of people affected [20] would make it likely that there is a significant unmet need in these population groups, but robust evidence for the effectiveness of targeted TB interventions is surprisingly scarce and urgently needed. Other underserved populations have been frequently mentioned as priority groups and TB control among these groups remains a challenge [15]. A key part of our survey was to identify perceived barriers to strategy implementation. Here, an important observation was that clinical, particularly tertiary services were felt to be prioritised compared with public health and prevention opportunities in some settings. While it is uncertain to what extent this represents the personal views of respondents, a perceived underprioritisation of public health services is cause for concern.

\section{Strengths and limitations}

The main strength of this study is that it achieved a 100\% response rate from TB programme leads or their delegates in the $31 \mathrm{EU} / \mathrm{EEA}$ countries. It can therefore be seen as a representative view of key TB control stakeholders in the EU/EEA, thereby allowing comparison with previous survey results [9]. Questions were 
Inadequate systems for ...

Rapid diagnostic testing

Infection control in healthcare facilities

Quality control within laboratories

Procuring and distributing laboratory supplies

Procuring and distributing TB drugs

Referring and transferring TB patients

TB control programme monitoring and evaluation

Timely and accurate surveillance information

Authority and accountability ...

Bureaucracy in wider healthcare system

Lack of leadership in healthcare system

Accountability for TB control programme targets

Leadership within national TB control programme

Communication issues between ...

Providers and recipients of healthcare

Healthcare and social care systems

Different levels of the healthcare system

Public health agency and clinical care providers

Need for training of ...

Surveillance scientists

Microbiologists/laboratory staff

Workforce shortages ...

Surveillance scientists

Microbiologists/laboratory staff

Specialist TB nurses

Specialist TB doctors

Funding issues in ...

Wider healthcare system

Facilities and healthcare for vulnerable population groups

Medical facilities in prisons

Laboratory services

National TB control and prevention programme

$$
\text { - }
$$

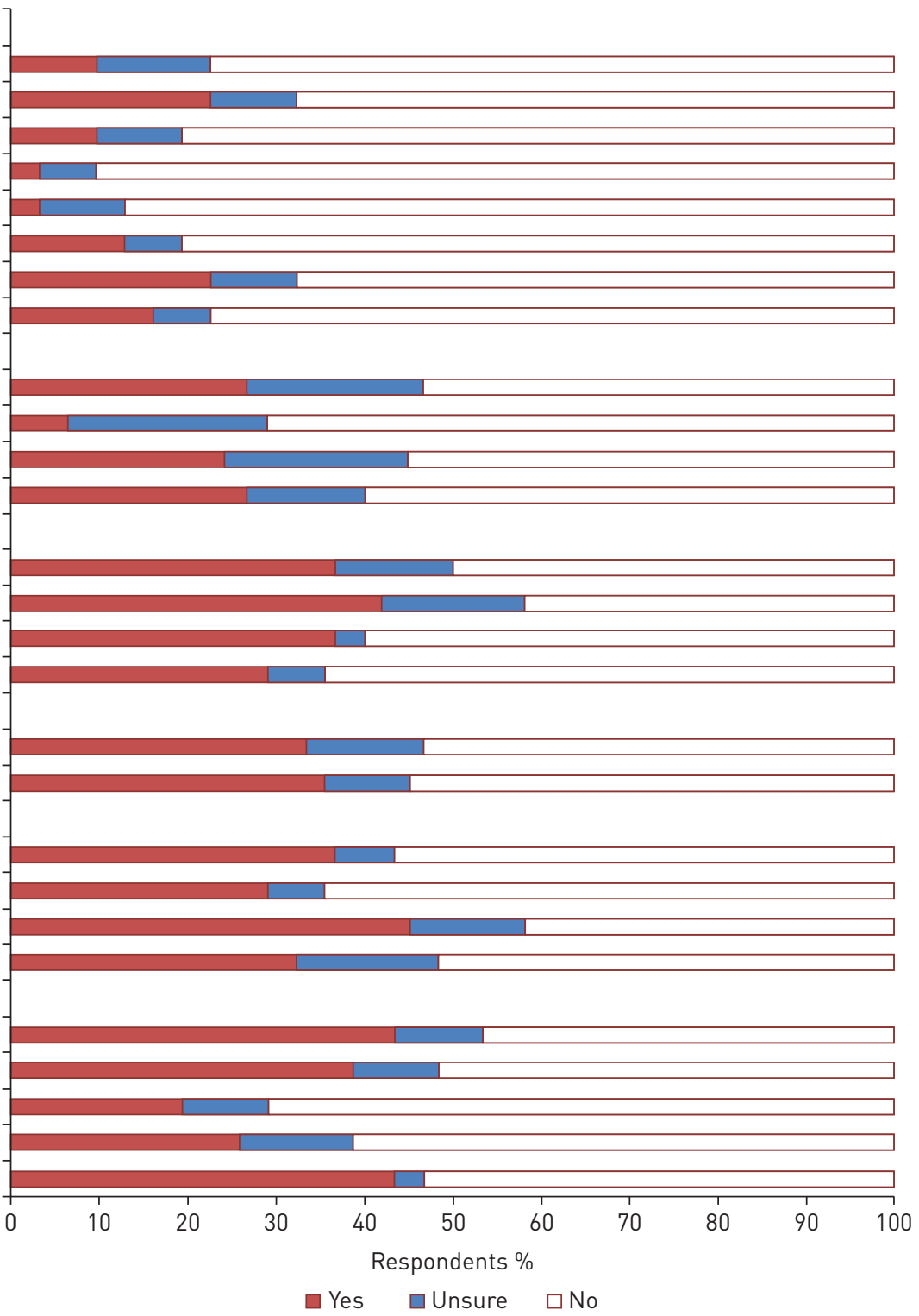

FIGURE 5 Barriers (health system constraints): “Which of the following factors impede TB control in your country?” TB: tuberculosis.

kept similar to validated frameworks and piloted among a small but key group of professionals, including two national TB programme leaders and the head of the ECDC TB Programme. The main limitation is that some responses may reflect the personal opinions of respondents, particularly responses to questions asking about priorities and barriers. We did not ask whether respondents had sought the views of colleagues, but we know that a number of respondents did consult within their programmes to provide correct and consensus responses to the survey. Although survey questions were worded in accordance with ECDC terminology [8], the survey was available only in English and linguistic differences might have caused ambiguities in the interpretation of questions. Also, some terms may overlap or mean different things in different countries (or to the same groups of people at different points in their journeys), e.g. asylum seekers, refugees and "documented"/"undocumented" migrants. To pre-empt these issues, telephone and e-mail support was provided for the duration of the survey and face-to-face at the 2017 Wolfheze Workshops. For the current article, we did not attempt an evidence synthesis based on free-text comments that accompanied some of the survey responses, but we did inspect any such comments to ensure the validity of our descriptive analyses and to provide additional detail where relevant.

Implications and recommendations for TB policy and practice in EU/EEA countries

Progress in the availability of national TB strategic plans has been slow, with half of EU/EEA countries not having a plan in place at the time of this survey despite publication of the WHO End TB Strategy in 2015 [6]. 
TABLE 3 Responses to selected questionnaire items in relation to national tuberculosis (TB) incidence $l \geqslant 10$ per 100000$)$

Countries
National TB control plan or strategy
Clearly defined organisational structure
Central coordination
Specific funding allocated to TB control
High-priority actions
Training and developing a specialist TB workforce
Introducing and implementing new tools for TB control
External quality assurance for laboratory services
Implementing electronic TB case registries
Staffing and expertise for national TB surveillance
Establishing or managing local TB control boards
Publishing and disseminating clinical guidelines
Raising awareness of TB at community/primary care level
Reaching vulnerable population groups
TB control in prisons
Latent TB infection screening in high-risk population groups
Screening for active TB in high-risk population groups
Ensuring continuity of TB drug supply
Screening for active TB in migrants from high-incidence countries
Contact tracing and outbreak investigation
BCG vaccination
MDR-TB
HIV/TB
Barriers to TB control and prevention
Recipients of care barriers (range 0-6)
Providers of care barriers (range 0-5)
Social and political constraints (range 0-6)
Health system constraints (range 0-27)

\section{1}

9 (42.9)

$13(61.9)$

$18(85.7)$

$2(28.6)$

$10(47.6)$

8 (38.1)

7 (33.3)

$12(57.1)$

9 (42.9)

$4(19.1)$

$6(30.0)$

8 (38.1)

17 (81.0)

8 (38.1)

$11(52.4)$

$13(61.9)$

$8(38.1)$

14 (66.7)

$13(61.9)$

$2(9.5)$

$10(47.6)$

7 (35.0)

$3(1-4)$

$2(1-3)$

$2(0-3)$

5 (2-7)
10

8 (80.0)

$10(100.0)$

10 (100.0)

$3(30.0)$

$5(50.0)$

$6(60.0)$

$5(50.0)$

$6(60.0)$

$3(30.0)$

1 (10.0)

$5(50.0)$

$5(50.0)$

7 (70.0)

$6(60.0)$

$4(40.0)$

$6(60.0)$

$6(60.0)$

3 (30.0)

$5(50.0)$

2 (20.0)

$8(80.0)$

7 (70.0)

3 (2-4)

$1.5(1-3)$

$1(0-2)$

$6.5(2-10)$

Data are presented as n, n (\%) or median (interquartile range). BCG: bacille Calmette-Guérin; MDR: multidrug-resistant.

Whether recent international meetings such as the Global Ministerial Conference on Ending TB in the Sustainable Development Era (Moscow, November 2017) and the UN High-Level Meeting on TB (New York, September 2018) will increase government commitment to, and prioritisation of, TB control and elimination across the EU/EEA remains to be seen. Clearly, having a plan is only the first step: implementation requires centralised coordination, sufficient funding and evidence-based interventions.

The EU/EEA has favourable indices for determinants of trends in TB incidence, such as economic growth, human development and public resources [21], and annual rates of decline for the region (4.3\% during the period 2007-2016) are faster than all other regions [1]. However, this downward trend is still unlikely to meet the WHO target of TB elimination by 2050 in European low-incidence countries [22]. A key issue with regard to recommendations for policy and practice in the EU/EEA is the considerable social, economic and epidemiological heterogeneity between and within countries. As our survey has shown, EU/ EEA countries that carry a high burden of $\mathrm{TB}$ in their native population, e.g. Romania accounted for almost one-quarter of reported cases in 2016, are understandably much less concerned about cases in foreign-born population groups than countries where these represent the vast majority of reported cases, e.g. 90\% in Sweden and 96\% in Malta [1]. However, commonalities (and common borders) exist that provide potential for EU/EEA-wide and local interventions. Several such areas were highlighted in the most recent ECDC/WHO TB monitoring and surveillance report for Europe [1], and it is instructive to match these with responses to our survey and with evidence for effective interventions.

Identifying and treating TB cases of foreign origin, and ensuring good access to healthcare for migrants and other vulnerable population groups, is clearly a priority in countries where these are foci for the majority of cases. Limited evidence for the effectiveness of interventions in vulnerable populations [16] and for active and latent TB screening in migrants $[23,24]$ should give impetus to rigorously conducted large-scale evaluations of different approaches to addressing this issue, given that any successful approach is likely to be generalisable across low TB incidence EU/EEA countries and many migrants cross internal EU/EEA borders in journeys from their ports of arrival. 
Prisons are a focus of higher TB and MDR-TB incidence in most countries [25]; although three-quarters of countries in our survey had a strategy for TB control in prisons, only half rated this as a priority area. Data on TB in prisons in EU/EEA countries is scarce, with only 18 countries providing monitoring data in the years to 2016 [1]. We echo the ECDC/WHO recommendation that all EU/EEA countries collect information to support accurate monitoring of TB in prisons at the EU/EEA level and, again, we would advocate for evaluations to provide an evidence base for interventions that are likely to be effective regardless of country.

Our survey highlighted a perceived need for investment in human resources/expertise. This indicates a need in higher TB incidence EU/EEA countries to expand specialist training for clinical staff, while low TB incidence countries can contribute collaboratively through guideline development, providing technical assistance, exchanging technology and strengthening research capacity. Indeed, cross-border collaboration between high and low TB incidence countries is one of eight priority action areas within the WHO/ European Respiratory Society framework towards TB elimination [26]. This will also address the issue in low TB incidence EU/EEA countries of clinicians having insufficient first-hand experience to manage TB cases, with $\mathrm{TB}$ being so rare in some countries that there is a danger of losing local knowledge and expertise [27].

"Inadequate systems for TB control programme monitoring and evaluation" was identified as a factor impeding TB control by only one-quarter of survey respondents, and three-quarters of countries had a strategy for monitoring and evaluation, yet the ECDC/WHO report indicated that only 14 out of 26 WHO targets could be effectively monitored based on data from EU/EEA countries, with reporting of LTBI, HIV status and treatment outcomes as areas requiring most improvement [1]. Monitoring EU/EEA-wide treatment outcomes is important given an apparent decline in success rates (from an average of $77 \%$ during 2011-2013 to 74\% in 2014-2015), substantial between-country variation, and success rates for both MDR- and XDR-TB that are far below WHO targets [1]. Benchmarking and identifying differences is essential if countries are to disseminate and share best clinical practice. At an epidemiological level, a common strategy enables monitoring of emerging threats, such as the increasing proportion of XDR-TB among MDR-TB cases (from $14 \%$ in 2012 to $21 \%$ in 2016) [1].

We note that routine collection of complete data from all countries for the wide range of indicators included in the ECDC/WHO report [1], which could be gradually expanded to collect data on, for example, palliative care for XDR-TB and comorbidities such as diabetes and mental health, largely obviates the need for future one-off surveys. In the meantime, we trust that our survey findings will serve to inform the development of an evidence-based toolkit that EU/EEA and other countries can use to design national TB strategies [11], thereby supporting these countries to work collaboratively towards TB elimination.

Acknowledgements: We would like to thank the respondents to the survey for their time and effort.

Author contributions: D. Zenner conceptualised the initial hypothesis and idea for the study and provided overall supervision. G. de Vries, K. Lönnroth and I. Abubakar contributed to the study concept and design. S.M. Collin and D. Zenner acquired the data and undertook the analyses. G.B. Migliori critically revised data interpretation and adaptation of the report to the manuscript format. S.M. Collin had full access to all the data in the study, and takes responsibility for the integrity of the data and the accuracy of the data analysis. All authors contributed to drafting the manuscript and reviewing it for important intellectual content. All authors approved the final manuscript.

Conflict of interest: None declared.

Support statement: This study is part of the E-DETECT TB project (709624) which has received funding from the European Union's Health Programme (2014-2020). The content of this article represents the views of the authors only and is their joint responsibility; it cannot be considered to reflect the views of the European Commission and/or the Consumers, Health, Agriculture and Food Executive Agency (CHAFEA) or any other body of the European Union. The European Commission and CHAFEA do not accept any responsibility for use that may be made of the information it contains. G.B. Migliori is under the operational research plan of the WHO Collaborating Centre for Tuberculosis and Lung Diseases, Fondazione S. Maugeri, Tradate, ITA-80, 2017-2020. Funding information for this article has been deposited with the Crossref Funder Registry.

\section{References}

1 European Centre for Disease Prevention and Control/World Health Organization Regional Office for Europe. Tuberculosis Surveillance and Monitoring in Europe 2018. Stockholm, ECDC, 2018.

2 Lönnroth K, Migliori GB, Abubakar I, et al. Towards tuberculosis elimination: an action framework for low-incidence countries. Eur Respir J 2015; 45: 928-952.

3 Rendon A, Fuentes Z, Torres-Duque CA, et al. Roadmap for tuberculosis elimination in Latin American and Caribbean countries: a strategic alliance. Eur Respir J 2016; 48: 1282-1287.

4 Voniatis C, Migliori GB, Voniatis M, et al. Tuberculosis elimination: dream or reality? The case of Cyprus. Eur Respir J 2014; 44: 543-546. 
5 Al Yaquobi F, Al-Abri S, Al-Abri B, et al. Tuberculosis elimination: a dream or a reality? The case of Oman. Eur Respir J 2018; 51: 1702027.

6 Uplekar M, Weil D, Lonnroth K, et al. WHO’s new End TB Strategy. Lancet 2015; 385: 1799-1801.

7 World Health Organization. Toolkit to Develop a National Strategic Plan for TB Prevention, Care and Control. Geneva, WHO, 2015.

8 European Centre for Disease Prevention and Control. Progessing Towards TB Elimination: A Follow-up to the Framework Action Plan to Fight Tuberculosis in the European Union. Stockholm, ECDC, 2010

9 D'Ambrosio L, Dara M, Tadolini M, et al. Tuberculosis elimination: theory and practice in Europe. Eur Respir J 2014; 43: 1410-1420.

10 Abubakar I, Matteelli A, de Vries G, et al. Towards tackling tuberculosis in vulnerable groups in the European Union: the E-DETECT TB consortium. Eur Respir J 2018; 51: 1702604.

11 The SURE Collaboration. SURE Guides for Preparing and Using Evidence-based Policy Briefs: 5. Identifying and Addressing Barriers to Implementing Policy Options. Version 2.1. 2011. https://epoc.cochrane.org/sites/epoc. cochrane.org/files/public/uploads/SURE-Guides-v2.1/Collectedfiles/sure_guides.html Date last accessed: September 29, 2017.

12 Broekmans JF, Migliori GB, Rieder HL, et al. European framework for tuberculosis control and elimination in countries with a low incidence. Recommendations of the World Health Organization (WHO), International Union Against Tuberculosis and Lung Disease (IUATLD) and Royal Netherlands Tuberculosis Association (KNCV) Working Group. Eur Respir J 2002; 19: 765-775.

13 Veen J, Migliori GB, Raviglione M, et al. Harmonisation of TB control in the WHO European region: the history of the Wolfheze Workshops. Eur Respir J 2011; 37: 950-959.

14 Migliori GB, Sotgiu G, Rosales-Klintz S, et al. ERS/ECDC Statement: European Union standards for tuberculosis care, 2017 update. Eur Respir J 2018; 51: 1702678.

15 de Vries SG, Cremers AL, Heuvelings CC, et al. Barriers and facilitators to the uptake of tuberculosis diagnostic and treatment services by hard-to-reach populations in countries of low and medium tuberculosis incidence: a systematic review of qualitative literature. Lancet Infect Dis 2017; 17: e128-e143.

16 Heuvelings CC, de Vries SG, Greve PF, et al. Effectiveness of interventions for diagnosis and treatment of tuberculosis in hard-to-reach populations in countries of low and medium tuberculosis incidence: a systematic review. Lancet Infect Dis 2017; 17: e144-e158.

17 Abid M, McCarthy N, Saldana L, et al. Extensively drug-resistant tuberculosis case in the Thames Valley, UK and public health interventions. J Infect Public Health 2011; 4: 207-210.

18 Gillini L, Centis R, D’Ambrosio L, et al. Is Europe ready to reach tuberculosis elimination? An outbreak report from Southern Italy. Eur Respir J 2015; 46: 274-277.

19 de Jong L, Pavlova M, Winters M, et al. A systematic literature review on the use and outcomes of maternal and child healthcare services by undocumented migrants in Europe. Eur J Public Health 2017; 27: 990-997.

20 International Organization for Migration. World Migration Report 2018. Geneva, IOM, 2017.

21 Suk JE, Manissero D, Buscher G, et al. Wealth inequality and tuberculosis elimination in Europe. Emerging Infect Dis 2009; 15: 1812-1814.

22 World Health Organization. Definitions and Reporting Framework for Tuberculosis. Geneva, WHO, 2013.

23 Greenaway C, Pareek M, Abou Chakra CN, et al. The effectiveness and cost-effectiveness of screening for active tuberculosis among migrants in the EU/EEA: a systematic review. Euro Surveill 2018; 23: 17-00542.

24 Greenaway C, Pareek M, Abou Chakra CN, et al. The effectiveness and cost-effectiveness of screening for latent tuberculosis among migrants in the EU/EEA: a systematic review. Euro Surveill 2018; 23: 17-00543.

25 Biadglegne F, Rodloff AC, Sack U. Review of the prevalence and drug resistance of tuberculosis in prisons: a hidden epidemic. Epidemiol Infect 2015; 143: 887-900.

26 World Health Organization. Framework Towards Tuberculosis Elimination in Low-incidence Countries. Geneva, WHO, 2014.

27 Luzzati R, Migliori GB, Zignol M, et al. Children under 5 years are at risk for tuberculosis after occasional contact with highly contagious patients: outbreak from a smear-positive healthcare worker. Eur Respir J 2017; 50: 1701414. 